\title{
Ultralow Loss Adiabatic Microring Resonator With THERMAL TUNING
}

\author{
A PREPRINT \\ Akhilesh S. P. Khope* \\ Microsoft Corporation \\ One Microsoft Way \\ Redmond, WA 98052 \\ *Work done while at UC Santa Barbara \\ akhkhope@gmail.com
}

June 6, 2021

\begin{abstract}
We report a micro-ring resonator with adiabatic bends, non contact waveguide heaters and small bend radius. The ring has the lowest reported off resonance loss and can support 8 wavelength division multiplexed channels at $200 \mathrm{GHz}$ spacing. We measure $0.49 \mathrm{~nm} / \mathrm{mW}$ tuning efficiency and $0.085 \mathrm{~dB}$ off resonance loss.
\end{abstract}

Keywords Microring Resonators · Photonic Integrated Circuits · Optical Switching Devices · Silicon Photonics

\section{Introduction}

Microring resonators (MRR) for the backbone of all silicon phootnics photoninc integrated circuits (PIC). High index contrast enables tight bend radius and high free spectral range (FSR) resonators. MRR off resonant loss or pass through loss at off resonant wavelengths is a major contributor to optical path loss when there are many MRRs along the path of light. We encounter this situation in switches [1, 2, 3, 4, 5, 6, 7, 8], add-drop multiplexers [9] and modulator arrays [10].

For a $N \times N$ switch, with L MRR at each crosspoint reported in [1] the worst case path loss of a switch scales linearly with L (number of MRR per crosspoint), the radix N and off-resonance loss of the MRR. Off-resonance loss of the MRR is the most important metric for the switch. Authors are a

MRR reported in literature do not have both low off-resonant loss and $>20 \mathrm{~nm}$ FSR required for wavelength division multiplexing (WDM) applications [11]. Second order filters are sensitive to fabrication variation. MRR where heater is in contact with the optical waveguide have higher optical loss and a higher tuning efficiency [11]. MRR filter with the lowest loss reported has a heater in the center and waveguide spokes connected to a microdisk. Optical loss of $0.05 \mathrm{~dB}$ is reported for this MRR in [12]. In this paper, we report our adiabatic microring resonator with adiabatic bends and coupling bus waveguide based on Bezier bends [13]. We report device design, experimental results and compare our MRR with other MRRs from literature.

\section{Methods}

Fig 1 (a) shows layout of the MRR. There inner edge of the MRR is an ellipse and outer edge a circle. The waveguide is wider at the point farthest from the bus waveguides similar to [11]. The outer radius is $4 \mu \mathrm{m}$ and semi-minor axis of the ellipse on the inner edge is $3.2 \mu \mathrm{m}$. The smallest gap between outer edge and and the bus waveguide is $0.18 \mu \mathrm{m}$. Minimum width of the MRR is $0.4 \mu \mathrm{m}$.

Heater dimentions are as follows, outer minor and major axis $3.8 \mu \mathrm{m}$ and $5.8 \mu \mathrm{m}$. Inner minor and major axis $3 \mu \mathrm{m}$ and $5 \mu \mathrm{m}$. The smallest gap between heater and MRR inner edge is $0.3 \mu \mathrm{m}$. Heater is p doped silicon waveguide. Fig. 2 (d) shows IV of heater. 
A PREPRINT - JUNE 6, 2021

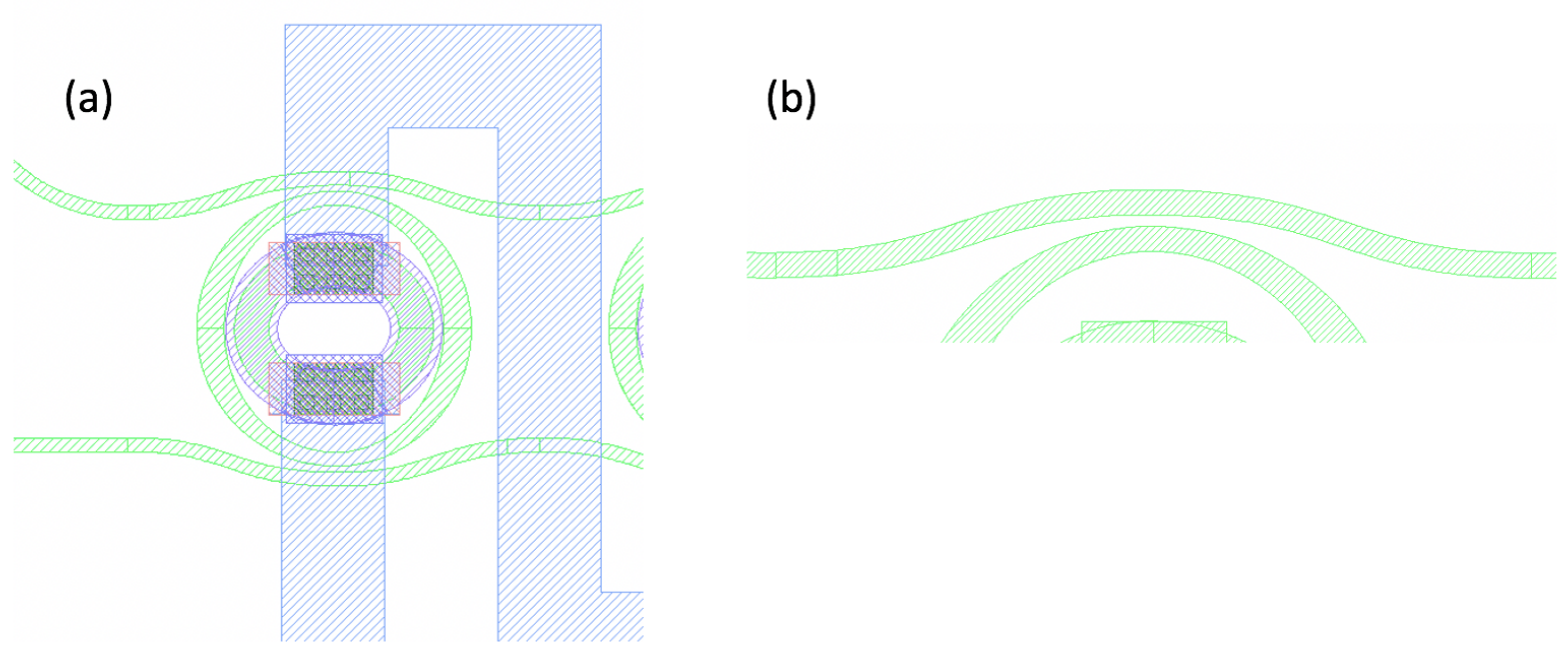

Figure 1: (a) Layout of the MRR (b) bezier coupler

Optical off resonance loss can be either due to scattering at the coupling region of MRR due to mode mismatch, optical loss in the path of light in the MRR due integrated heater or mode overlap of propagating mode with $\mathrm{p}$ doped heater.

We use Lumerical 3D FDTD to design bus waveguide to MRR coupling. The bus waveguide near the MRR is a bezier curve and reduces scaterring loss. Fig 1 (b) shows the bezier coupler. The bezier secion from input waveguide to minimum width point is $6 \mu \mathrm{m}$ and height of the top of the bezier curve from the straight input routing waveguide is $1.4 \mu \mathrm{m}$. We used parameter sweeps using for loops to minimize the extinction at the through port of the MRR.

\section{Experimental Results annd Discussions}

In this section, we present experimental results of the MRR. Fig 2 (a) shows the drop and through spectrum of the MRR. Extinction of the MRR at through port is $<-15 \mathrm{~dB}$. Fig 2 (b) shows tuning of the MRR by $16 \mathrm{~nm}$ out of $25.6 \mathrm{~nm}$ FSR. Fig 2 (c) shows tuning efficiency of the MRR $=0.49 \mathrm{~nm} / \mathrm{mW}$. We measure off resonance loss of $0.085 \mathrm{~dB}$. This off resonance loss is half the off resonance loss of the MRR ring designs from [14] and measured in [4].We measured this loss by taking an average loss measured with test structures with 0,10, 20 and 40 MRRs in series. We use functions from Lumos a python library for our measurements [15]. In the following table, we summarize work from several papers. We consider MRRs with $>20 \mathrm{~nm}$ FSR. The tuning efficiency reported in this work is much higher than tuning efficiency reported in other papers because of non contact heaters.

\begin{tabular}{||ccccc||}
\hline Device & $\begin{array}{c}\text { Tuning } \\
\text { Efficiency }\end{array}$ & FSR & $\begin{array}{c}\text { Switching } \\
\text { Speed }\end{array}$ & Loss (dB) \\
\hline MRR with adiabatic bends [11] & $4.4 \mu W / G H z$ & $32.85 \mathrm{~nm}$ & $1 \mu s$ & - \\
\hline $\begin{array}{c}\text { Micro-disk resonator } \\
\text { with integrated heater }[12]\end{array}$ & $5.5 \mu W / G H z$ & $35 \mathrm{~nm}$ & - & 0.05 (drop) \\
\hline Second order MRR [14] & $-* *$ & $19 \mathrm{~nm}$ & - & - \\
\hline This work & $15.75 \mu W / G H z(0.49 \mathrm{~nm} / \mathrm{mW})$ & $25.6 \mathrm{~nm}$ & - & $0.09 \mathrm{~dB}$ (off loss) \\
\hline
\end{tabular}

** The authors report tuning efficiency of $0.69 \mathrm{~nm} / \mathrm{mW}$ (per ring). We do not report this value in the table. They calculate this value based on $36 \mathrm{~mW}$ per FSR tuning. The paper does not report full FSR tuning and IV curves of MRR are non linear as shown in Fig. 2 (d). The authors do not mention how they extrapolate the tuning power. We calculate $0.49 \mathrm{~nm} / \mathrm{mW}$ tuning efficiency based on $16 \mathrm{~nm}$ tuning range instead of the whole FSR. 

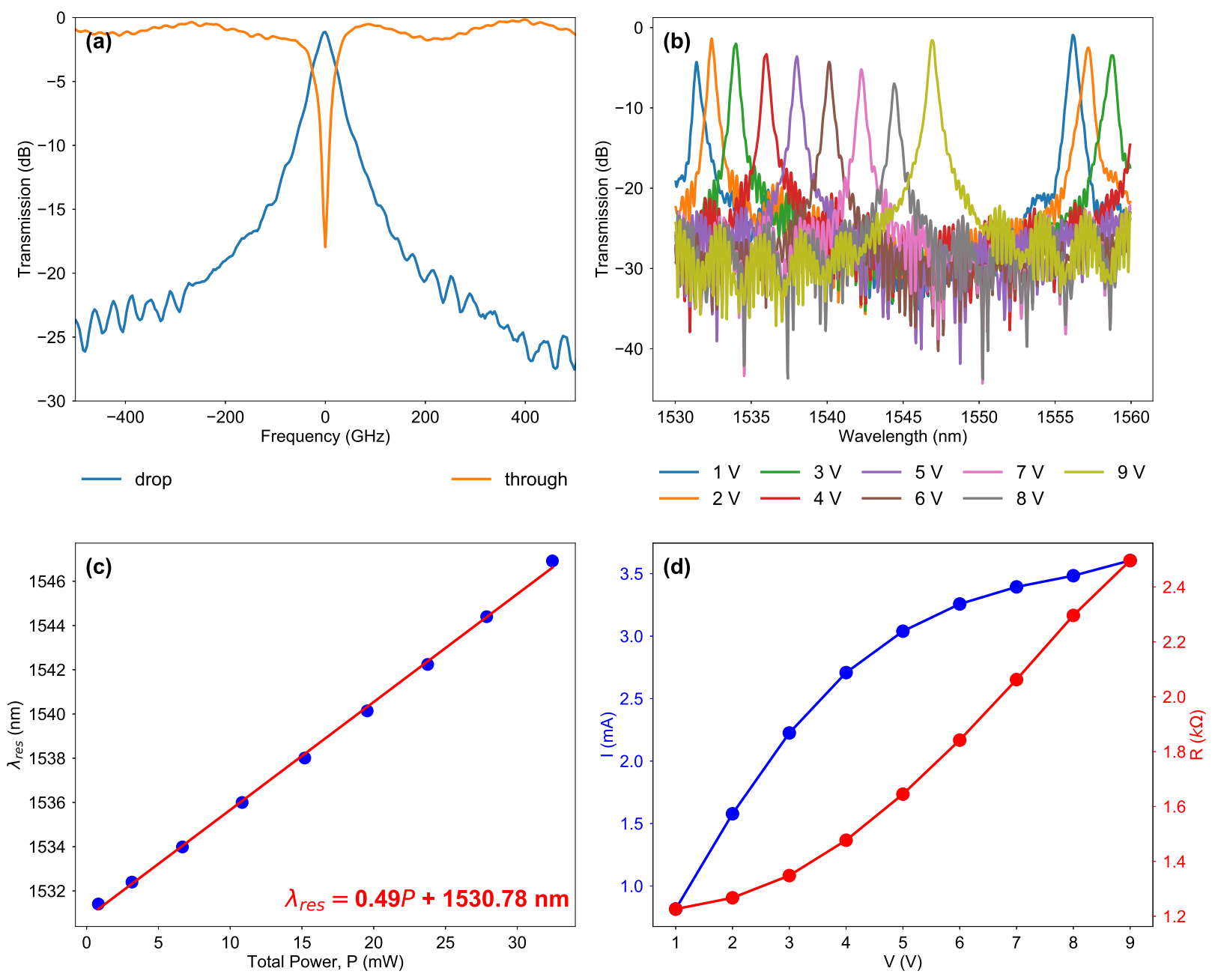

Figure 2: Measurements on custom first order ring resonator (a) Drop and through transfer spectra of a single first-order ring resonator, (b) Tuning curve of custom ring,(c) Tuning is demonstrated. The ring tunes by $16 \mathrm{~nm}(\mathrm{FSR}=25.6 \mathrm{~nm})$ with power efficiency of $0.49 \mathrm{~nm} / \mathrm{mW}$. (d) IV and RV curves for the cascaded ring resonators. Nonlinear dependence of $\mathrm{R}$ vs. $\mathrm{V}$ is measured.

\section{Conclusion}

We report a MRR with adiabatic bends and coupler optimized for low scatering loss. Our MRR is suitable for use in WDM systems with 4 channels at $400 \mathrm{GHz}$ spacing or 8 channels at $200 \mathrm{GHz}$ spacing based on $16 \mathrm{~nm} / 25.6 \mathrm{~nm}$ tuning range. This MRR has the lowest reported measured off resonance loss among all the MRR papers in literature and is the most suitable for WDM applications.

Acknowledgement: The author thanks AIM Photonics for funding this work.

\section{References}

[1] Akhilesh SP Khope, Mitra Saeidi, Raymond Yu, Xinru Wu, Andrew M Netherton, Yuan Liu, Zeyu Zhang, Yujie Xia, Garey Fleeman, Alexander Spott, et al. Multi-wavelength selective crossbar switch. Optics express, 27(4):5203-5216, 2019.

[2] Akhilesh S. P. Khope, Takako Hirokawa, Andrew M Netherton, Mitra Saeidi, Yujie Xia, Nicolas Volet, Clint Schow, Roger Helkey, Luke Theogarajan, Adel A. M. Saleh, et al. On-chip wavelength locking for photonic switches. Optics letters, 42(23):4934-4937, 2017. 
[3] Roberto Proietti, Xian Xiao, Marjan Fariborz, Pouya Fotouhi, Yu Zhang, and Ben SJ. Flex-lions: A silicon photonic bandwidth-reconfigurable optical switch fabric. IEICE Transactions on Communications, 103(11):1190-1198, 2020.

[4] Akhilesh S. P. Khope, Songtao Liu, Zeyu Zhang, Andrew M. Netherton, Rebecca L. Hwang, Aaron Wissing, Jesus Perez, Franklin Tang, Clint Schow, Roger Helkey, Rod C. Alferness, Adel A. M. Saleh, and John E. Bowers. $2 \lambda$ switch. Opt. Lett., 45(19):5340-5343, Oct 2020.

[5] Akhilesh SP Khope, Roger Helkey, Songtao Liu, Sairaj Khope, Rod C Alferness, Adel AM Saleh, and John E Bowers. Scalable multicast hybrid broadband-crossbar wavelength selective switch: proposal and analysis. Optics Letters, 46(2):448-451, 2021.

[6] Yishen Huang, Qixiang Cheng, Anthony Rizzo, and Keren Bergman. High-performance microring-assisted space-and-wavelength selective switch. Optical Fiber Communication Conference (OFC), pages 1-3, 2020.

[7] Andrew W Poon, Xianshu Luo, Fang Xu, and Hui Chen. Cascaded microresonator-based matrix switch for silicon on-chip optical interconnection. Proceedings of the IEEE, 97(7):1216-1238, 2009.

[8] Akhilesh SP Khope, Anirban Samanta, Xian Xiao, Ben Yoo, and John E Bowers. Review of integrated photonic elastic wdm switches for data centers. arXiv preprint arXiv:2105.14934, 2021.

[9] Noam Ophir, Christopher Mineo, David Mountain, and Keren Bergman. Silicon photonic microring links for high-bandwidth-density, low-power chip i/o. IEEE Micro, 33(1):54-67, 2013.

[10] Po Dong, Robert Gatdula, Kwangwoong Kim, Jeffrey H Sinsky, Argishti Melikyan, Young-Kai Chen, Guilhem De Valicourt, and Jeffrey Lee. Simultaneous wavelength locking of microring modulator array with a single monitoring signal. Optics express, 25(14):16040-16046, 2017.

[11] Michael R Watts, William A Zortman, Douglas C Trotter, Gregory N Nielson, David L Luck, and Ralph W Young. Adiabatic resonant microrings (arms) with directly integrated thermal microphotonics. In Conference on Lasers and Electro-Optics, page CPDB10. Optical Society of America, 2009.

[12] Erman Timurdogan, Zhan Su, Jie Sun, Michele Moresco, Gerald Leake, Douglas D Coolbaugh, and Michael R Watts. A high-q tunable interior-ridge microring filter. In 2014 Conference on Lasers and Electro-Optics (CLEO)-Laser Science to Photonic Applications, pages 1-2. IEEE, 2014.

[13] Jiwen Zhang. C-bézier curves and surfaces. Graphical Models and Image Processing, 61(1):2-15, 1999.

[14] CL Manganelli, P Pintus, F Gambini, D Fowler, M Fournier, S Faralli, C Kopp, and CJ Oton. Large-fsr thermally tunable double-ring filters for wdm applications in silicon photonics. IEEE Photonics Journal, 9(1):1-10, 2017.

[15] Akhilesh SP Khope. Lumos: A python instrument control library for photonics. 2021. 